\title{
Calidad de la democracia y desacuerdos fácticos. Una exploración parcial a la relación entre ciudadanos, políticos y expertos ${ }^{1}$
}

\author{
DANTE AVARO \\ Doctor en Filosofía (Universidad Nacional Autónoma de México) \\ Investigador del Consejo Nacional de Investigaciones Científicas y Técnicas, Argentina \\ dante.avaro@7tres.biz
}

\begin{abstract}
Resumen El creciente rol del conocimiento y el actuar de los expertos en las democracias contemporáneas pueden ser abordados en tres niveles bien delimitados: 1) la distribución del conocimiento, los actores que conocen (expertos y especialistas) y su encastre en la legitimidad democrática constituyen el nivel epistémico de la democracia;2) el rol que se le atribuye al conocimiento, al experto y las reglas de la expertise en los funcionamientos democráticos configura el nivel de la calidad de la democracia; y finalmente, 3) cómo usan los Estados y gobiernos el conocimiento experto para mejorar los funcionamientos democráticos conforma el nivel de agenda de la calidad de la democracia. Este trabajo se ubica en el segundo nivel, es decir, entre el nivel filosófico-normativo y el empírico-informativo. Este recorte del objeto de estudio está justificado por dos razones: a) la discusión filosófica podría avanzar más en la medida que los debates tengan acceso a información sistematizada y validaciones empíricas, sin embargo b) los estudios empíricos encuentran, a mi modo de ver, una limitante a su desarrollo, éste está estancado por cierta imprecisión en la definición conceptual de "experto" y su rol en la teoría democrática. Este trabajo tiene como propósito analizar este último asunto.
\end{abstract}

Palabras clave: calidad de la democracia, desacuerdos fácticos, expertos, ciudadanos.

\section{Introducción}

"Do you not know, my son, with how little wisdom the world is governed?”. Axel Gustafsson Oxenstierna, Conde de Södermöre $(1583-1654)^{2}$.

T a relación entre la política y el conocimiento se remonta al inicio Lde la filosofía política. Las metáforas náuticas griegas, en que expertos timoneles llevan navíos desde el mar bravío a la tranquila playa (tanto para fundar, abastecer o re-fundar la polis), nos heredan un amplio campo de composición entre auctor [Augere], doxa y episteme que perdura hasta

1 Una presentación parcial de este trabajo se realizó en ACEVAL en la Semana Internacional de la Evaluación, Casa Reyes Heroles, Ciudad de México, 18 de junio de 2015.

2 Ver King $(1887,40)$. Encontré este pasaje de la carta enviada por el conde a su hijo John durante 1648 en una cita de F. Von Hayek a su trabajo "El ideal democrático y la contención del poder". 
nuestros días. Recientemente ha tomado impulso, de nueva cuenta, el debate normativo sobre el rol del conocimiento y la expertise ${ }^{3}$ en las democracias contemporáneas. La ubicación más difundida de este debate osciló entre justificaciones deontológicas y consecuencialistas de la democracia, es decir, entre los procedimientos y las consecuencias (ver Greppi, 2013). Sin embargo, la cuestión ya no parece ser aquella en que el conocimiento y la expertise sirven o pueden servir para justificar la democracia, sino que tanto el conocimiento y el trabajo experto constituyen a la democracia. Estlund (2003 y 2009) y Christiano (2009 y 2013) introducen al debate una argumentación normativa que posiciona a la democracia con un valor intrínseco que tendencialmente la conduce a tomar buenas decisiones ${ }^{4}$. De tal forma que la autoridad normativa contiene en sí misma un nivel epistémico que hace que la democracia, como régimen político, produzca o genere buenas decisiones. Dicho de otra forma: la democracia es valiosa y se legitima también por la tendencia ha producir o generar decisiones correctas. Estos debates normativos ni han estado aislados en una turris eburnea, ni sus impactos en el mundo político han consistido en la típica esterilidad de arar el mar, por el contrario han atravesado de múliples formas y en diferentes niveles la evolución de las modernas democracias representativas.

Desde el inicio del siglo XIX con la aplicación de la estadística a la comprensión relacional entre educación y delito (Cullen, 1975 citado en Wiess, 2009, 7) hasta la propuesta reciente del ex primer ministro británico Tony Blair de promover una política pública basada en la evidencia (ver Head, 2010), las democracias fueron generando diferentes hechos institucionales que conforman un entramado de capas geológicas diversas que dan cuenta de la utilización del conocimiento (y de su relevancia) en las decisiones autoritativas. Desde la perspectiva de los funcionamientos institucionales de la democracia resulta imprescindible conocer cómo se estructura la oferta y la demanda de conocimiento entorno a la construcción de evidencia científica para la hechura de las políticas (ver Carden, 2009). Recientemente se ha avanzado bastante en describir los modos o formas de ofertar conocimiento (ver Campbell-Pedersen, 2011 y 2014), pero se requiere avanzar más en la cuestión de cómo el Estado y los gobiernos demandan conocimiento y a los expertos que lo actualizan (ver Garcé, 2014). Sin embargo, a pesar de contar con una tijera marshalliana incompleta entorno a la demanda-oferta de activos intangibles intransferibles (conocimiento-expertise), este hecho no ha paralizado a la ciencia política, en especial los estudios sobre los funcionamientos democráticos, que se los han ingeniado, por un lado, para resaltar la importancia creciente y decisiva del entramado institucional intensivo en conocimiento-expertise para la calidad democrática (ver Stein et. al., 2006), y por otro, utilizando la expertise para caracterizar los funcionamientos democráticos, su calidad y dimensión productiva (dimensión de resultados democráticos) $)^{5}$.

Los cambios organizacionales al interior del Estado acaecidos desde los años ochenta a escala mundial (ver Boltanski-Chiapello, 2005) renovaron el interés por la relación entre políticos y administradores impulsando no sólo una nueva agenda de investigación sobre el servicio civil de carrera (burocracia), sino que ampliaron el espectro de investigaciones en torno a la agenda de la calidad del gobierno (ver Prewitt et. al., 2012). Estudios sobre el funcionamiento institucional de los bancos centrales, agencias reguladoras, comisiones asesoras, consejos consultivos, etc. (ver Rosanvallon, 2009) fueron engrosando la base empírica en dos sentidos diferentes pero complementarios. Por un lado, dando cuenta de las limitaciones y carencias, en términos de conocimiento y expertise, que poseen los Estados para intervenir en problemas y temas cada vez más técnicos y complejos, y de la necesidad creciente de los gobiernos de solicitar colaboración a las diferentes organizaciones de ciudadanos para construir problemas públicos y sus soluciones (ver Doubleday-Wilsdon, 2013). Por otro lado, la aceptación gubernamental de la existencia de la "sociedad del riesgo" (Beck, 2002), el "hecho de la expertise" (Holst-Molander, 2014, 14), y finalmente la innovación tecnológica que inmersa en la sociedad y economía del conocimiento mueve constantemente las interacciones políticas y abre nuevas perspectivas de legitimación democrática no visibilizadas hasta el momento a nivel de las instituciones y de los diseños democráticos (ver Jassanoff, 2011). De este modo, la llamada agenda de la gobernanza, por un lado, y la esfera de "legitimidad reflexiva" (Rosanvallon, 2009) de la democracia, por otro, comenzaron a ser herramientas conceptuales muy utilizadas para retratar estas nuevas capas en la interacción entre ciudadanos, políticos y expertos de las sociedades democráticas contemporáneas (ver Innes-Booher, 2003).

Los lentes conceptuales de la gobernanza y la esfera de legitimidad reflexiva han corrido en forma paralela a la expansión democrática en plena globalización.

3 Si bien el Diccionario de Americanismos de la Asociación de Academias de la Lengua Española recoge la entrada "experticia", prefiero seguir utilizando expertise por considerar que aquella no es un sustituto adecuado para las complejidades teóricas y empíricas en donde la expertise se inserta (ver Fischer, 2009). [Nota: con respecto al género tomo en cuenta el femenino de "la" experticia y por tanto utilizo "la/una" expertise]. 4 Véase también Sunstein (2006), Estlund (2009) y Landemore-Elster (2014).

5 Me refiero a los interesantes debates al interior de la agenda de investigación de la calidad democrática sobre la utilización de la opinión de expertos para construir índices o métricas de calidad (ver Munck, 2002 y 2010). 
Esto permitió contar con más estudios enfocados a delimitar las capacidades de los Estados, sus limitaciones técnicas, a fomentar la utilización de nuevos métodos de recolección, almacenamiento y publiceidad de las evidencias para el diseño y la evaluación de las políticas, mayor interacción entre ciudadanos, políticos y públicos sobre las políticas y sus agendas (ver Geoff, 2013; Sabel-Zeitlin, 2012). Permitió, además, el estudio del entramado institucional de las agencias reguladoras en las reformas estructurales llevadas a cabo en muchos países durante los años ochenta y noventa. Sin embargo, por una de arena, hay otra de cal. La mayoría de los estudios comparados sobre la calidad del gobierno y buena gobernanza están enfocados en la estabilidad de las políticas, los resultados, o bien en los diseños institucionales de las agencias reguladoras, pero se indaga poco o casi nada en cómo los Estados y los gobiernos utilizan el conocimiento y la expertise, cómo se designan los expertos, con qué criterios se toman decisiones expertas en las agencias independientes, cómo se supervisa, quién lo hace, cómo se solicita la rendición de cuentas sobre el trabajo experto ${ }^{6}$. Por otra parte, cuando las investigaciones hacen énfasis en los errores técnicos de las decisiones autoritativas de las democracias como es el caso de las crisis financieras, las supervisiones bancarias, los resultados negativos en materia de calificación de riesgo financiero, los escándalos tipo Enron, Baring Brothers, subprime, por citar los más conocidos que han atrapado a los públicos globalizados, la función de los expertos queda algo diluida entre dos extremos: o están inmersos en lo que Galbraith (2004) ha denominado "la economía del fraude inocente" o como actores de un mundo que tiene que lidiar con el riesgo. Es decir, los errores técnicos de las decisiones autoritativas o son decisiones técnicas que buscan satisfacer intereses privados en pos de los públicos, es decir, se entremezclan y se confunden con la agenda investigativa sobre la corrupción (ver Stiglitz, 2003a, 2003b y 2010; Sapir, 2004), o bien son el resultado de un largo proceso en que Fausto le ganó la partida a Prometeo, lo que implica que la técnica y los expertos han desatado fuerzas que ya no pueden controlar (ver Sibilia, 2005). El nivel filosófico de la última y el reductivo de la primera han obrado como una pinza que promueve una falta de reflejos teóricos y conceptuales en la ciencia política para analizar una cuestión central de la vida democrática: cuánta relevancia tienen los expertos en las decisiones autoritativas y cómo los ciudadanos pueden llegar a identificar a esos expertos. Resumiendo, suponiendo normativamente que los ciudadanos se muestran interesados en la calidad de las decisiones públicas, al tiempo que creen que las democracias producen mejores decisiones que otros regímenes políticos, entonces aparece una tensión de dificil resolución para las democracias que requieren legitimar la expertise con la equidad política en las decisiones autoritativas. Esta discusión normativa, que re-edita la histórica tensión entre la expertise frente a la equidad política, puede ser mejor servida en la medida que contemos con estudios empíricos comparados de cómo los gobiernos utilizan el conocimiento y la expertise. Sin embargo para que los estudios empíricos puedan alimentar la discusión sobre el nivel epistémico de la democracia se requiere contestar dos preguntas: 1) cómo los ciudadanos pueden reconocer a los expertos, y 2) qué mecanismos o diseños institucionales resultan efectivos para que los expertos intervinientes sean expertos que mediante su actividad competente produzcan mejores resultados que cualquier otro régimen.

Por las limitaciones propias de este trabajo me apoyo en dos premisas. La primera, en una democracia de calidad los ciudadanos, en caso de requerirlo, tienen que contar con la posibilidad de reconstruir públicamente la influencia de la actividad experta tanto en las decisiones autoritativas como en el procesamiento de los desacuerdos fácticos. Segunda, como los desacuerdos doxásticos y fácticos están vinculados de múltiples formas, en una democracia de calidad los ciudadanos pueden desear discutir públicamente los mecanismos de compatibilización entre equidad política y expertise utilizados para legitimar las políticas (actos autoritativos). Este trabajo está orientado a analizar conceptualmente la cuestión de quién es un experto, los mecanismos de reconocimiento de la expertise y su vinculación con la teoría democrática $(\mathbb{S} .2)$. Propongo un marco analítico para explorar cómo los ciudadanos pueden en una democracia identificar a los expertos (\$.3).Y si bien resulta imprescindible contar con un marco analítico para identificar a los expertos, es necesario que se articule hacia adelante con la cuestión de la rendición de cuentas, y hacia atrás con los espacios instituciones en dónde interactúan (\$.4). Finalmente concluyo con los aportes que el sendero consecuencualista puede hacer a los estudios de la calidad de la democracia.

\section{Equidad política y expertise.}

Ante la dificultad de incorporar la ciencia en
la política se suele utilizar una respuesta que
se le atribuye a Albert Einstein ante el hecho
relativamente asombroso de separar el átomo,
pero la imposibilidad manifiesta de controlar
la política nuclear, a lo cual Einstein supues-
tamente sostuvo: "That is simple, my friend:
because politics is more difficult than physics". 
Abrir la democracia al conocimiento y a los expertos ha engendrado, desde siempre en los pensadores, un peligro latente: el pasaje a la epistocracia, es decir, enfrentar el peligro de pasar de un gobierno de iguales a un gobierno de expertos. Los demócratas saben que el valor democrático por excelencia radica en haber construido un régimen político en que la elección de fines corresponde a la esfera doxástica, no epistémica, y saben también que las decisiones políticas tienen una dimensión normativa relevante, y que en ese nivel no hay ni puede haber expertos morales. El demócrata sabe que del lado de la ecuación 'equidad política' no puede producirse una expertise moral y aunque como ha dicho Robert Dahl (1989) si bien esto no quiere decir que todo lo referido a los desacuerdos morales sea una cuestión de gustos o preferencias, no hay expertos en moral porque no hay un método válido y aceptable para todos ${ }^{7}$.

Sin embargo, los ciudadanos no sólo son diferentes (el fact of pluralism de Rawls), sino que además tienen diferentes dotaciones de conocimientos, habilidades, destrezas, competencias que da como resultado que algunos tengan más autoridad epistémica que otros en diferentes áreas de la vida pública. Aún si los demócratas que habitan las actuales democracias no desearan, además de valorar y defender la equidad política, dar buenos resultados o generar decisiones autoritativas correctas, por ejemplo más atractivas que decisiones tomadas al azar, requerirían aún compatibilizar el conocimiento/expertise con el valor normativo de la equidad política (Dahl, 1989 y 2008), dado que nuestras democracias están inmersas en el "fact of experti$s e "$ (Holst, 2014a). En las democracias los ciudadanos, como población anónima que ortorga legitimidad a los actos autoritativos, no son expertos. Esto significa que la equidad moral ejercida por medio del principio de la equidad política no los convierte en expertos morales. Sin embargo, reconocen que hay individuos que poseen autoridad epistémica sobre ciertos asuntos públicos. Por tanto, los demócratas son conscientes que las modernas democracias compatibilizan, aunque no siempre sepan como, equidad política y conocimiento para permitir disfrutar de ciertos niveles de calidad democrática, i.e. que la democracia produzca buenos resultados. De alguna forma los ciudadanos perciben que en las democracias modernas hay dos esferas que reclaman para sí cierta autonomía, pero que finalmente interactúan ${ }^{8}$. Compatibilizar, aquí, es un verbo que se conjuga en dos niveles: 1) dotar de legitimidad al trabajo de los expertos en las decisiones autoritativas de la democracia, y 2) discriminar (en el sentido de cribar) cuándo estamos en presencia de intercambios bloqueados (no permitidos) entre el dominio de la expertise y el de la equidad política. Si para evitar la epistocracia es necesario asumir que el dominio de la política y el de la expertise son esferas diferenciadas, y que una de ellas no debe ejercer una influencia bloqueada sobre la otra, todavía queda el asunto de mayor importancia apenas intuido: cómo ha de darse cuenta el ciudadano cuando la técnica somete a la política, o cuando ésta politiza a la primera, es decir, cuándo existe una interacción no permitida entre equidad política y la expertise.

Si la filosofia política teme el deslizamiento de la democracia a la epistocracia, la sociología (política) acuñó conceptualmente el término tecnocracia para dar cuenta del predominio de los expertos y técnicos en las decisiones públicas. Concepto que no gozó ni goza de buena reputación en las ciencias sociales, amargo pero sin llegar a ser una cantarella para las democracias contemporáneas, es más invocado que utilizado para describir los entresijos de las decisiones autoritativas de las modernas democracias. Acompañó el devenir del siglo XX y tuvo una reaparición fulgurante tras la caída del Muro de Berlín, la expansión globalizadora y los procesos de reformas estructurales market friendly. Pero al nivel que he decidido mantenerme en este trabajo uno de los mayores desafios no consiste en esclarecer por qué razones la tecnocracia tiene mala fama, sino cómo un ciudadano puede estar seguro que está en presencia de ella, es decir, en una interacción no permitida. En algunas ocasiones, como por ejemplo en las críticas realizadas al Banco Mundial y al Fondo Monetario Internacional, la tecnocracia aparece como una forma degenerativa de la utilización del conocimiento (ver Sapir, 2004); en otras, fundamentalmente en las críticas a la independencia de los bancos centrales, aparece como un calificativo denostativo de la expertise. Sin embargo, el sabor amargo de la tecnocracia en estos casos puede relacionarse con diferentes raíces. En el primer ejemplo, con la presunción que los expertos se identifican con ciertos intereses privados y los satisfacen a costa del interés público, en el segundo caso, con una usurpación a la actividad política (nivel doxástico) y de los políticos. Como se puede observar en el Diagrama 1 los escenarios epistocráticos se conforman cuando los políticos tienen (tienden a) una escasa o nula intervención en los

7 Aquí desacuerdos morales sirven, desde la perspectiva dahliana, como una etiqueta general e intercambiable con el uso que aquí le doy a los desacuerdos doxásticos.

8 Los resultados proporcionados a la pregunta "Having Expert Make Decisions" confeccionada por la World Values Survey resultan sumamente ilustrativos 
actos autoritativos $)^{9}$. Este sabor amargo parece desaparecer cuando la democracia toma una poción mágica que se puede poner en estos términos: La técnica si no está al servicio de los políticos se convierte en tecnocracia, es decir, en mala técnica. De otra forma, desde la perspectiva de los políticos (y de ciertas posiciones doxásticas) hay intercambios bloqueados que se permiten en la medida que colaboren con sus aparentes intereses (que serían a su vez los mismos que los de sus representados). Lo que quiere decir que la política cuando politiza a la ciencia y a los expertos puede resultar beneficiosa en la medida que los intereses de los ciudadanos y de los políticos coincidan. Sin embargo esta forma extrema de visualizar la relación entre políticos y expertos, i.e. la tecnocracia, deja a los ciudadanos en medio de un verdadero galimatías: 1) como los ciudadanos no pueden saber, justamente porque no son expertos, si los expertos actúan guiados por la expertise o por sus intereses personales (ver Holt-Molander, 2014, 18) pueden llegar a aceptar un intercambio bloqueado entre políticos y expertos, esto es, que la política politice a la ciencia y a los expertos en la medida que los intereses de los ciudadanos y los políticos no divergan. Sin embargo, 2) en la medida que los intereses de éstos últimos comienzan a distanciarse frente a los actos autoritativos, los ciudadanos pueden desear la aparición pública de unas voces imparciales e independientes que encarnadas en los expertos puedan poner en perspectiva los asuntos fácticos que atraviesan a las decisiones autoritativas que llevan adelante los políticos (ver Arimoto-Sato, 2012). En esta vena las cosas se pueden poner más confusas para los ciudadanos. Puede suceder que la ausencia de voces imparciales e independientes se deba no sólo a que los políticos politizaron a la ciencia y a la expertise, sino a que los políticos gobiernan sin evidencia, orientados sólamente por la intuición, astucia, instinto o la mera orientación ideológica.

Las esferas autónomas de la política por un lado y del conocimiento / expertise por otro no necesariamente funcionan como la última retaguardia ante la amenza de la expertise a la equidad política, la empalizada de la democracia siempre está amenazada por el acecho de la epistocracia. Esta relación entre equidad política $\mathrm{y}$ expertise fundada en compartimentos estancos y relacionados mediante intercambios bloqueados genera más dudas que certezas al ciudadano sobre la influencia, por un lado, del experto sobre los actos autoritativos y, por otro, sobre el tipo de decisiones que toman los políticos en función de la evidencia científica disponible. El modelo de separación entre equidad política y expertise en esferas autónomas, distantes y reguladas por intercambios bloqueados expone lo complejo del asunto de manera efectivamente sencilla: por un lado, evitar el gobierno de los expertos sobre la política, y por otro, propender a que los dogmatismos y las decisiones irracionales no eviten utilizar las evidencias científicas disponibles (Pedersen, 2014). Sin embargo, si los ciudadanos esperan que los políticos actúen de manera honesta y decidan con la mejor evidencia científica de la que pueden disponer, no está claro para él que los expertos propongan y que los políticos decidan, o que éstos decidan todo el tiempo a espaldas de lo que sugiere la expertise. Como hemos visto supra hay cierta permisividad a los intercambios bloqueados por temor a que los expertos decidan, y por otro lado, la amenaza tecnocrática no es razón suficiente para que los políticos eviten el uso de la evidencia científica en los actos autoritativos.

Si bien los casos extremos (nula y máxima intervención de los políticos en los actos autoritativos descrita en el Diagrama 1) son situaciones lógicamente posibles, resulta altamente probable que la mayoría de los actos autoritativos de las democracias contemporáneas se encuentren a mitad camino de estos extremos (zona sombreada del Diagrama 1). Quizá sea más complejo, pero también más realista, pensar a las dos esferas autónomas, pero comunicadas; antes que compartimentos estancos, como esponjas que permean flujos, como un múltiple complejo de membranas que permiten circular al tiempo que filtrar intercambios y que poseen unos plugins que sin cambiar la lógica sistémica propia de cada esfera van generando conectores exteriores para comunicarse e intercambiar información. En este sentido la relación entre equidad política y expertise ya no viene marcada por los intercambios bloqueados ni por la usurpación de fronteras, sino más bien por tratar de escudriñar que tanta influencia ejercen los expertos en los actos autoritativos y de qué manera utilizan los políticos las evidencias científicas para fundar sus decisiones autoritativas [Vid Diagrama 2]. Visto desde esta perspectiva resulta relevante para la calidad democrática analizar cómo los ciudadanos identifican a los expertos sobre todo porque ellos no lo son.

9 Lo interesante de los escenarios epistocráticos no radica en observarlos como un corrimiento de un régimen democrático a otro (epistocrático), sino en la potencialidad para observar áreas de gobierno y sus decisiones autoritativas dentro del régimen democrático pero que tendrían características epistocráticas. Y al nivel que me muevo aquí no interesa saber si son espacios que han cedido los políticos o son espacios usurpados, para los ciudadanos lo relevante es que emergen como espacios manejados tecnocráticamente. 


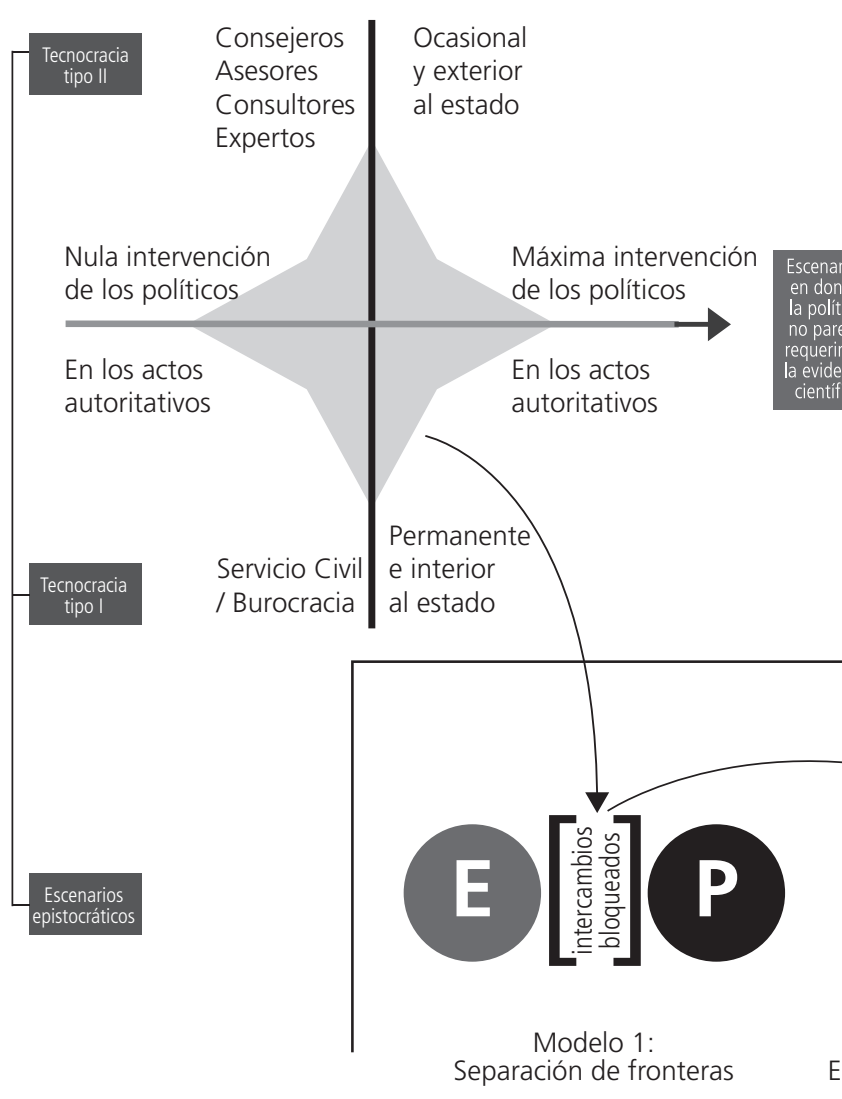

I

1

1

Consejeros

$\begin{array}{ll}1 & \text { Asesores } \\ \text { Consultores }\end{array}$

Expertos

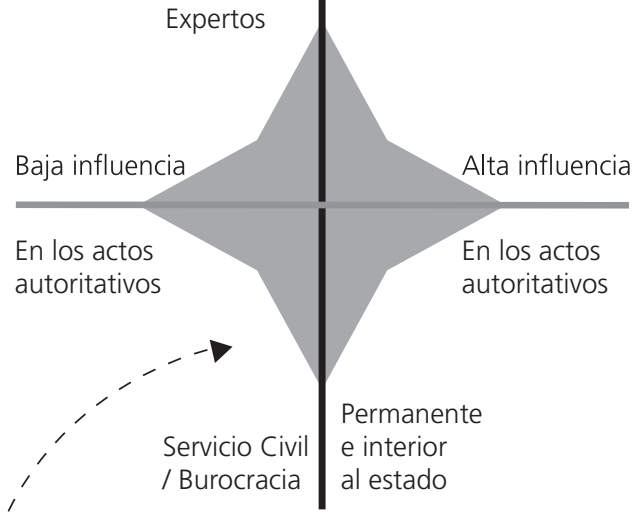

Fuente: elaboración propia

\section{El sendero consecuencialista.}

“¿Quién decide cuando los médicos no están de acuerdo?”. Alexander Pope (1688-1744)

La agenda de la calidad de la democracia no ha ignorado que la dimensión "resultados" (Morlino, 2004) es fundamental para analizar la calidad de una democracia. 'Resultados' aquí puede interpretarse como el balance de actos autoritativos producto de decisiones correctas (buenas decisiones). Si bien la incorporación de la dimensión resultados en los estudios sobre la calidad de la democracia ha promovido un debate sobre la propia definición de la democracia, dicha discusión no ha requerido plantear nuevos atributos ni motivaciones a los demócratas que la habitan. Aunque la ciencia política asume, dentro de una arraigada tradición ${ }^{10}$, que los ciudadanos tienen doxa pero no episteme, supuesto que en cierta medida se mantiene porque rara vez los ciudadanos ponen en tela de juicio que las instituciones democráticas operan sobre la base de que la delegación de los procesos de decisión a los expertos es apropiado y legítimo (ver Holst-Molander, 2014, 16), no resulta teóricamente contradictorio con la posibilidad empírica que entre ciudadanos, políticos y expertos existan diálogos entorno a los desacuerdos fácticos que atraviesan ciertos actos autoritativos, fundamentalmente con aquellos que emergen con fuerza en la agenda pública de los diversos públicos democráticos. En este sentido, intentar dar respuesta, por un lado, a qué tanta influencia ejercen los expertos en los actos autoritativos y cómo, por otro, utilizan el conocimiento y la expertise los políticos no requiere considerar atributos y motivaciones ciudadanas diferentes a las que el corpus teórico de la agenda de la calidad democrática ya le atribuye. No se requiere incorporar nuevos atributos (episteme), ni motivaciones ad hoc a los ciudadanos para postular que las democracias de calidad funcionan incorporando las evidencias científicas tanto a los debates públicos como a los procesos decisorios, y que consideran a las decisiones que ignoran a las evidencias científicas como defectuosas (ver Pedersen, 2014). Sin embargo, si los ciudadanos carecen de episteme cómo reconocen a los expertos, y aún si los políticos le dijeran: "-éstos 
son los expertos”. ¿Los ciudadanos pueden discrepar al respecto? Por tanto si los ciudadanos tienen ciertas dificultades, en su condición de no-experto (layperson), en reconocer quiénes son los expertos, entonces cómo pueden darle la autoridad a lo que dicen y hacen (ver Goldman, 2011) ${ }^{11}$.

Los ciudadanos además de los desacuerdos de índole doxástica pueden experimentar desacuerdos no-normativos (ver Kappel, 2014), esto es, sobre hechos. Este tipo de desacuerdos no-normativos son sobre la verdad (falsedad) de una proposición factual (supongamos $p^{\star}$ ). Para simplificar, aquí supongo que los ciudadanos mantienen desacuerdos fácticos al menos por tres tipos de mecanismos: i) los ciudadanos pueden tener principios epistémicos incompatibles, ii) pueden confiar en diferentes autoridades epistémicas, y iii) pueden interpretar las evidencias de manera diferente y alternativa (ver Kappel, 2014). Para que los ciudadanos tengan desacuerdos fácticos no es preciso, en este enfoque, atribuirle episteme a los ciudadanos, basta solamente que exista libertad de ideas, circulación de información, libertad de prensa y la libertad de constituir múltiples espacios de diálogo, discusión e interpelación públicos. En el mundo real resulta dificil separar los desacuerdos fácticos de los doxásticos (Kappel, 2014), en muchas ocasiones se complementan, incluso se lo puede describir como de co-pertenencia. Estos dos tipos de desacuerdos son irreductibles e ineliminables en las democracias contemporáneas, los doxásticos son abordados en la agenda de la calidad democrática bajo el andamiaje teórico-analítico-conceptual del enfoque procedimental, mientras que los desacuerdos de índole fáctica podrían ser analizados desde la perspectiva del sendero consecuencialiasta que a continuación presento.

No todo desacuerdo fáctico atraviesa actos autoritativos, y no todo acto autoritativo refleja de manera automática en la opinión pública los desacuerdos fácticos que lo perforan. En una democracia que goce de las más amplias libertades aceptables se espera que los ciudadanos mantengan una variedad amplia y de dificil sistematización de desacuerdos fácticos, sin embargo sólo algunos de ellos constituyen un tratamiento político democrático, puesto que sobre éstos la autoridad pública legislará o implementará políticas. Así el universo de desacuerdos fácticos, desde la perspectiva de la agenda de la calidad de la democracia, queda reducida a aquellos que constituyen la evidencia discutible y en disputa para los actos autoritativos (Vid Etapa I.0 Diagrama 3). Siguiendo el trabajo de Kappel (2014) propongo el siguiente marco analítico para presentar e identificar una disputa fáctica: existe un desacuerdo fáctico entre el ciudadano $\mathrm{A}\left(c_{\mathrm{a}}\right)$ y el ciudadano $\mathrm{B}\left(c_{\mathrm{b}}\right)$ toda vez que, (a) $c_{\mathrm{a}}$ y $c_{\mathrm{b}}$ no están de acuerdo sobre qué opción de política $(O P)$ es mejor o preferida; (b) existe una proposición fáctica $p^{\star}$ que produce que sus ordenamientos o preferencias de políticas difieran entre sí, y finalmente (c) en el caso que $c_{\mathrm{a}}$ y $c_{\mathrm{b}}$ tuviesen la misma actitud doxástica frente al asunto $p$ entonces (a) desaparece del plano doxástico durante el tiempo de coincidencia o convergencia de actitud doxástica. Esta manera de presentar los desacuerdos fácticos entre ciudadanos permite:

1. Relacionar los desacuerdos fácticos con actos autoritativos $(\mathrm{OP})$, es decir, los desacuerdos fácticos que se deben tomar en cuenta en la agenda de la calidad de la democracia son los que atraviesan a los actos autoritativos.

2. Que son justamente los desacuerdos fácticos sobre $\mathrm{p}$ los que constituyen diferentes ordenamientos de políticas. Esto vincula la apreciación que hacen los ciudadanos sobre las evidencias con autoridades epistémicas (expertos) y los probables desacuerdos. Pero además abre la posibilidad de relacionar la evaluación de las evidencias que hacen los ciudadanos sobre $\mathrm{p}$ con los ordenamientos de políticas y con los espacios institucionales en donde se procesan los desacuerdos entre expertos (ver Etapa I.1. Diagrama 3). De forma tal que los espacios institucionales y los expertos no son neutrales en tanto autoridad epistémica frente a los desacuerdos fácticos de los ciudadanos y sus ordenamientos de políticas. Así se va formando un entramado complejo entre expertos y ciudadanos que opera no sólo al nivel de los desacuerdos fácticos, sino también en los desacuerdos doxásticos por medio del ordenamiento de políticas entre los ciudadanos.

3. Supongamos que sobre un hipotético asunto $p$ los ciudadanos tienen una (im)probable y similar postura doxástica, por la cláusula (c) desaparece el desacuerdo sobre las opciones de política [cláusula (a)]. Sin embargo este caso no afirma ni niega que exista la posibilidad de que los ciudadanos no mantengan desacuerdos fácticos sobre $\mathrm{p}^{\star}$ solamente nos dice que frente al acto autoritativo no hay registro en la opinión pública de esos desacuerdos. Puede suceder que los ciudadanos no tengan (suficientes) referencias epistémicas para estar en desacuerdo, que los expertos no han participado, que las instancias de diálogo entre ciudadanos y expertos han sido insuficientes, que el gobierno no se ha preocupado en reunir evidencia científica, que los ciudadanos estaban ocupados en otras cuestiones o bien que no les interesa, entre otras situaciones posibles. Pero como los ciudadanos no sólo son soberanamente caprichosos votando, sino también conformando cambiantes públicos existe la posibilidad que frente a los escenarios futuros del 
acto autoritativo emerjan desacuerdos fácticos ex post. Un demócrata preocupado por la calidad de su democracia quizá experimente la necesidad de contar con la posibilidad de reconstruir el diálogo con los expertos y los políticos, revisando de nueva cuenta las evidencias y la opción de política. De tal forma que el esquema propuesto coloca a los desacuerdos fácticos no sólo al inicio del camino, sino también como una forma de desandarlo, juzgar y evaluar los actos autoritativos emprendidos por el gobierno, lo que para un demócrata comprometido con la transparencia, la rendición de cuentas y la responsabilidad resulta atractivo para asegurar la calidad democrática.

Lo que para el ciudadano se presenta como ordenamientos de opciones de políticas, es decir, un amasijo de posturas doxásticas, interpretaciones diferentes y alternativas sobre las evidencias, discursos epistémicos alternativos (ver Etapa I.1. en el Diagrama 3), para el analista es pasible de separación y reconstrucción por etapas, lo que aquí se sugiere bajo el nombre de sendero consecuencialista. Un ejemplo hipotético puede llegar a esclarecer mejor este asunto. Supongamos un país y su banco central más o menos independiente tal y como son descritos en la literatura reciente. Este país está atravesando un leve proceso inflacionario, a los demócratas como se sabe no le gustan los procesos inflacionarios porque destruyen la moneda social. Comenzó a circular en los diferentes públicos que conforman la opinión pública la idea que el banco central, en medio de ese contexto inflacionario, aumentará la base monetaria. Incrementar la base monetaria es $p^{\star}$ frente al asunto público $p$ (leve proceso inflacionario). Los ciudadanos orientarán sus opciones de política (política monetaria expansiva o contractiva) dados los desacuerdos que mantengan entorno a $p^{\star}$, pero sus valoraciones y juicios sobre $p^{\star}$ dependerán, dado que no son expertos, sobre lo que discutan, dialoguen y crean las autoridades epistémicas, por ejemplo los economistas. Supongamos que los economistas de este hipotético país ante la pregunta "La inflación es primariamente un fenómeno monetario" piensan lo siguiente: un $22.8 \%$ está de acuerdo, un $28.8 \%$ está de acuerdo pero con reservas, y un 45.9\% está en desacuerdo, el resto no contesta ${ }^{12}$. Si los economistas de este ejemplo están, como comunidad epistémica en medio de un desacuerdo, es altamente probable que al interior del espacio institucional encargado de tomar la decisión autoritativa (el banco central) también lo estén. Un demócrata puede pensar en que la manera de comunicar y procesar los desacuerdos fácticos por parte de los espacios institucionales y los expertos no sólo influye en los desacuerdos fácticos que mantengan los ciudadanos, sino también en las opciones de políticas preferidas, además de transparentar los procesos decisorios. Finalmente, el ciudadano como no-experto delega y confia en que las instituciones democráticas utilizarán de la mejor manera posible el conocimiento y el trabajo experto para producir las mejores decisiones posibles.

Cuando los desacuerdos fácticos, las disputas epistémicas, las controversias sobre la interpretación de las evidencias que atraviesan a los actos autoritativos resultan fácilmente referenciables a espacios institucionales específicos (p.e. el banco central) resulta más fácil para el ciudadano identificar a los que fungen bajo el rol de expertos. Sin embargo, no siempre resulta asequible que los ciudadanos tengan la posibilidad de identificar con claridad ni los espacios institucionales ni a los expertos que interactúan al interior de ellos. Los espacios institucionales pueden ser formales, pero también informales. Internos al Estado y de carácter permanente, como los bancos centrales o agencias reguladoras. Pero también pueden ser periféricos al Estado y temporalmente acotados, como comisiones asesoras ad hoc. Pueden ser de carácter contingente y puntal como consultorías especializadas. La existencia de espacios institucionales visibles y transparentes puede ser deseable tanto desde la perspectiva del ciudadano como del analista, puesto que los espacios institucionales no sólo son puntos nodales de referenciamiento para los desacuerdos doxásticos, sino que brindan pistas sobre cómo se designan los expertos que procesan esos desacuerdos. Los espacios institucionales, desde la perspectiva de la agenda de la calidad democrática, también brindan información sobre la interacción entre procedimiento y episteme. ¿Cómo se designan a los expertos? ¿Cómo se procesan los desacuerdos? ¿Cómo se discuten las evidencias? Resultan preguntas claves para describir los procedimientos institucionales, el trabajo de los expertos y los actos autoritativos. Conocer cómo funciona esta zona de solapamiento entre procedimientos y episteme puede resultar de mucha importancia para enjuiciar la calidad democrática, sin embargo conocer cómo se designan a los expertos no contesta a la pregunta básica del ciudadano: Dada su condición de no-experto cómo reconoce al experto y cómo sabe si los expertos usan sus competencias, destrezas y habilidades en el sentido correcto (ver Holst-Molander, 2014, 14).

\section{Expertos, instituciones y rendición de cuentas.}

\footnotetext{
"La reputación es una vida imaginaria en la vida de los demás; una cosa fuera de nosotros antes de nuestra muerte." Alexander Pope (1688-1744)
} 
Diagrama 3: Sendero consecuencialista

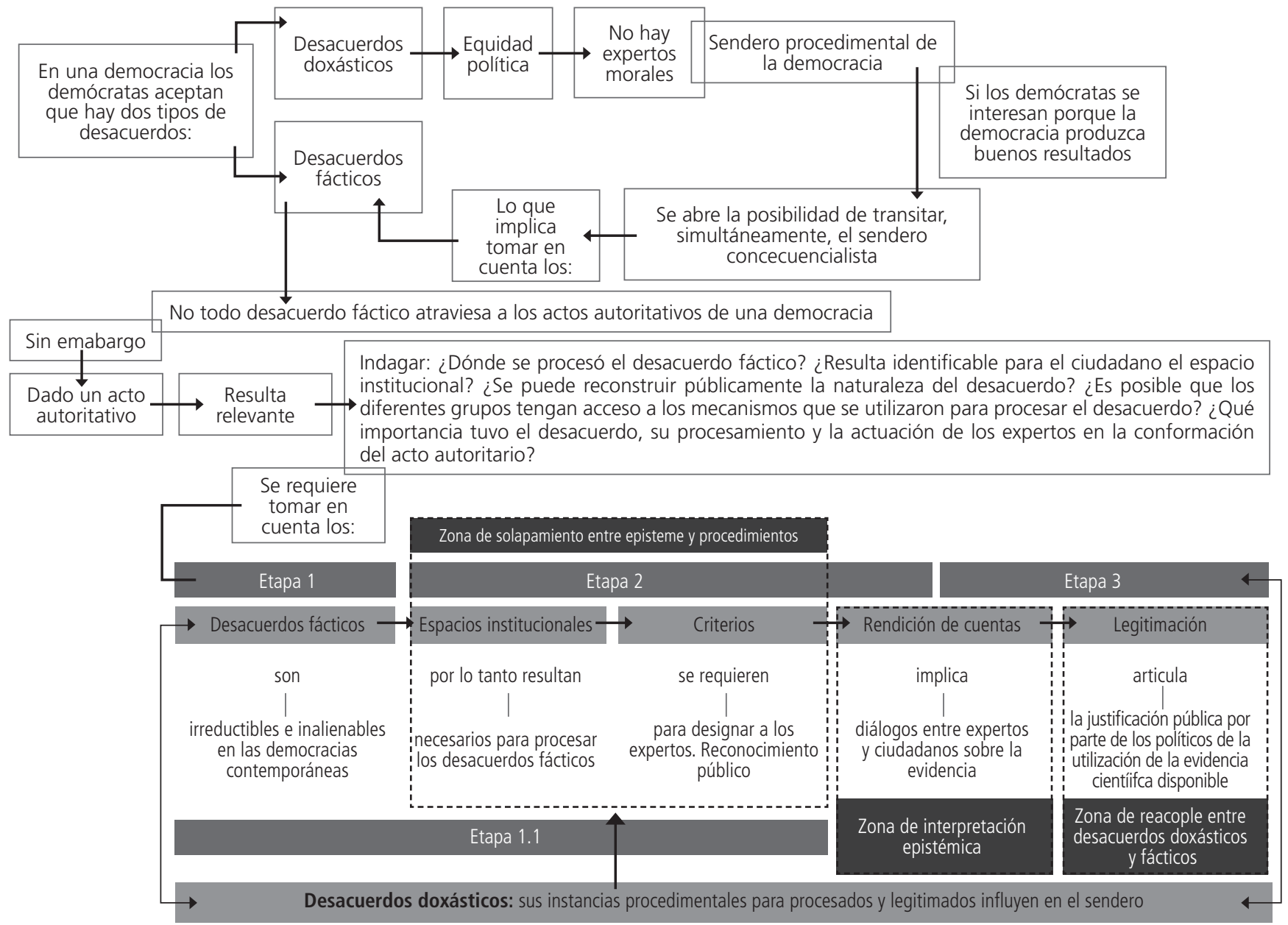

Fuente: elaboración propia

En las democracias actuales los expertos que intervienen en los distintos procesos que culminan en actos autoritativos son designados por medio de alguno de estos tres criterios:

1. Las autoridades electas mediante el voto popular designan a expertos, que a su vez designan a expertos, y éstos designan a otros, en una cadena $n$ de delegación, en donde $n$ implica los grados de complejidad epistémica que la expertise debe enfrentar de cara al asunto público a resolver.

2. Las autoridades electas mediante voto popular designan a expertos mediante criterios que el Legislador o el Constituyente han establecido de forma antecedente, autorizándolos en muchos casos a realizar actos autoritativos.

3. Los expertos ocupan dentro de una comunidad de expertise cargos mediante criterios que el Legislador y/o el Constituyente han establecidos previamente y que los autoriza a producir actos autoritativos.

El criterio (c) es un subgénero del (b). Además tanto en (b) como en (c) los expertos pueden designar expertos en $n$ niveles de delegación. Identificar los criterios de designación de expertos dice algo que ya sabíamos: que a nivel procedimental los ciudadanos aceptan o consienten la participación de expertos en la conformación de los actos autoritativos. Sin embargo, conocer cómo se designan los expertos no resuelve el problema de fondo de los ciudadanos, cómo reconocer a los expertos, es decir, como tener certeza que no son impostores que en un hipotético caso extremo no sean más que falsos expertos morales que se escabullen en los intersticios de los desacuerdos fácticos para imponer sus visiones doxásticas. Los expertos designados mediante los criterios (b) y (c) suelen ser, en la mayoría de las democracias contemporáneas, aprobados por comisiones parlamentarias mixtas, lo que le induce pensar al ciudadano que los parlamentarios sopesarán trayectorias profesionales, opiniones de otros expertos, capacidades e historiales laborales referidos a la expertise, sin embargo los demócratas saben que en esos procesos de designación se introducen, también, otros elementos de negociación entre fuerzas políticas y objetivos partidarios. Si bien las comisiones mixtas parlamentarias funcionan como un filtro que selecciona y transparenta las designaciones de expertos no responden a la cuestión primordial que se plantean los ciudadanos como no expertos: los expertos actuarán guiados por su expertise o influenciados por otros 
intereses. La cuestión de cómo reconocer la expertise no resulta fácilmente eliminable.

Goldman (2011) ha afirmado que un experto es un profesional que posee más proposiciones verdaderas y tiene menos creencias falsas que otros individuos en un determinado campo de saber y dominio de hacer ${ }^{13}$. Esta definición es relativamente potente:

1. Un experto no tiene todo el tiempo y para todos los asuntos de su campo de saber y de hacer proposiciones verdaderas, sino un mix entre aciertos y equívocos. Esto incorpora el asunto de márgenes de errores cognitivos de los expertos, errores cognitivos de la comunidad epistémica de referencia y las relaciones humanas al interior del conocimiento institucionalizado (ver Fischer, 2009). En síntesis: el experto puede fallar.

2. Identificando de esta forma al experto ya no resulta tan relevante para el ciudadano indagar qué tipo de intereses tienen los expertos con el tema o asunto que requiere de su opinión o intervención, puesto que un experto puede tener algún tipo de identificación o interés personal en el asunto y estar en lo correcto, o puede ser totalmente desinteresado y estar equivocado (ver Holst-Molander, 2014, 22). Por tanto, lo relevante para el ciudadano es que el experto tenga más proposiciones verdaderas que creencias falsas sobre el asunto público en cuestión.

3. Un experto no sólo tiene que parecer, sino que también tiene que serlo. Resumiendo: el historial (track records) reputacional del experto es un indicador de la calidad de la expertise.

Suponiendo las demás dimensiones que integran la calidad democrática ceteris paribus, el demócrata puede creer que una democracia tendrá más calidad en la medida que los espacios institucionales encargados de procesar los desacuerdos fácticos transparenten los criterios de designación de expertos, los identifiquen y seleccionen en función del criterio imparcial del historial reputacional. Esta interacción entre procedimientos y episteme no sólo mejora la confianza de los ciudadanos en los expertos y sus actividades, sino que existen mayores oportunidades de obtener un mejor nivel de calidad en el asesoramiento y en el tratamiento de las evidencias para la toma de decisiones autoritativas, es decir, más chances de que la democracia produzca mejores decisiones. Pero también al identificar a los expertos mediante el historial reputacional se incrementan los márgenes para que los políticos tengan más libertad al buscar el asesoramiento en el momento oportuno, asegurar la mayor independencia, perspectivas amplias y equilibradas y un manejo ex ante de incertidumbres y riesgos (ver Arimoto-Sato, 2012, 1176-1177). Sin embargo, queda pendiente la cuestión de cuánta influencia ejercen los expertos en los actos autoritativos.

Si el historial reputacional se convierte en un pilar fundamental para identificar a los expertos la cuestión de cuánta influencia ejercen en los actos autoritativos puede quedar acotada a la rendición de cuentas en un sentido amplio como diálogos entre expertos, ciudadanos y políticos. La rendición de cuentas es una forma de poner a prueba el historial reputacional frente a cada nuevo evento público que requiera la intervención de la expertise y en este sentido la rendición de cuentas funciona en el terreno clásico de legitimar tanto la participación de los expertos como los resultados (al nivel de no-expertos y expertos) y por otro lado, la rendición de cuentas opera como una forma de poner límites al poder ejercido por el experto sobre el no-experto ( $\mathrm{y}$ opera en la relación entre múltiples expertos con los no-expertos). Sin embargo, apuesto aquí por la etiqueta más difusa y débil de 'diálogo' que de 'rendición de cuentas' en sentido tradicional. Lo hago por las siguientes razones:

1. La rendición de cuentas requiere acotar un particular dominio de decisión-problema (ver Ross, 1973 citado en Steets, 2010, 17), quizá esclarecer cuál es el dominio particular de decisión-problema es parte del trabajo encargado a los expertos.

2. No siempre resulta claro identificar en los espacios institucionales que actúan los expertos quién es el principal de los expertos considerados éstos como agentes. Puede suceder que estemos frente a un escenario de múltiples principales, con intereses encontrados, que elevan los costos de información y monitoreo y que redunde en la imposibilidad de una acción colectiva de supervisión.

3. Aunque se pudiera identificar el principal no resulta fácil cómo sancionaría a los expertos que sugieren, aconsejan, pero que no toman la última decisión. Menos aún como una posible e hipotética sanción podría influenciar en los criterios independientes e imparciales que todo experto debería tener dado que esos atributos constituyen su historial reputacional y por eso es consultado.

4. La intervención de expertos en un asunto público puede requerir de la colaboración de múltiples expertos, provenientes de diferentes culturas epistémicas, lo que produce dos problemas: a) el clásico asunto de "varias manos" (problem of many hands propuesto por Thompson, 1980), y también b) diferentes formas de entender la rendición de cuentas, culturas organizacionales diversas e incluso estándares contradictorios, lo que Koppel (2005) ha

13 Tomando en cuenta la variedad de perspectivas analizadas en Ericsson et. al. (2006) la postura adoptada por Goldman (2011) resulta sencilla y adaptable a un número bastante extenso de expertise. 
denominado "multiple accountabilities disorder" (citado en Steets, 2010, 32 y ss.).

Resulta altamente probable que un ciudadano que acepta la intervención de los expertos en las decisiones autoritativas no pueda conocer con cierto detalle el grado de influencia que los expertos tienen en las decisiones y la conformación de las políticas, puede suceder que el criterio de historial reputacional no funcione adecuadamente para la elección de los expertos, sin embargo en la medida que los espacios institucionales encargados de procesar los desacuerdos fácticos sean transparentes e imparciales en el sentido descrito supra y funcionen de manera incipiente los diálogos entre expertos, ciudadanos y políticos, el ciudadano, en su condición de no-experto, podría pensar que la democracia goza de más calidad que si ninguno de esos mecanismos estuviera funcionando.

\section{Conclusión}

Los desacuerdos fácticos, como categoría analítica, funcionan como un articulador entre: i) los distintos ordenamiento de políticas de los ciudadanos con los actos autoritativos, al tiempo que ii) describe las interacciones entre el trabajo de los expertos, como soporte de la utilización de la evidencia científica de las políticas, con las diferentes valoraciones e interpretaciones que mantengan los ciudadanos. Esto se produce, según expuse en el $\$ .2$, sin necesidad de imputarle nuevos atributos y comportamientos a los ciudadanos a los ya contemplados por el enfoque predominante en los estudios sobre la calidad democrática. Lo que permite analizar la dimensión resultados de la calidad democrática desde su interior y no como un (contingente) resultado, sino como un proceso que relaciona procedimientos con espisteme, es decir, abordando la cuestión de oferta de expertise con la utilización del conocimiento para diferentes y múltiples actos autoritativos, lo que focaliza la dimensión resultados de la democracia en la interacción entre equidad política y episteme. La identificación de los expertos en el plano de los espacios institucionales, criterios de designación e historial reputacional permiten detener el lente observacional no tanto en los grados de influencia de los expertos sobre los actos autoritativos y sobre sus intereses, sino en los diseños institucionales que aquí fueron retratados exploratoriamente como diálogos entre ciudadanos, expertos y políticos.

\section{Referencias}

ARIMOTO, T.; SATO, Y. Rebuilding public trust in science for policy-making. Science and Society, v. 337, n. 6099, p. 1176-1177, 2012.

BECK, U. La sociedad del riesgo global. Madrid: Siglo XXI, 2002.

BOLTANSKI, L.; CHIAPELLO, E. The new spirit of capitalism. Londres:Verso, 2005.

CAMPBELL, J. L.; PEDERSEN, O. Knowledge regimes and comparative political economy. En: BÉLAND, D.; COX, R. H. (eds.). Ideas and politics in social science research. Oxford: Oxford University Press, 2011.

CAMPBELL, J. L.; PEDERSEN, O. The national origins of policy ideas: knowledge regimes in the United States, France, Germany, and Denmark. Princeton, NJ: Princeton University Press, 2014.

CARDEN, F. Del conocimiento a la política. Barcelona: Icaria, 2009.

CHRISTIANO, T. Estlund on democratic authority. The Journal of Political Philosophy, v. 17, n. 2, p. 228-240, 2009.

CHRISTIANO,T. Rational deliberation among experts and citizens. En: PARKINSON, J.; MANSBRIDGE, J. (eds.). Deliberative systems: deliberative democracy at the large scale, Cambridge: Cambridge University Press, 2013.

CULlEN, M. J. The statistical movement in early Victorian Britain: the foundations of empirical social research, Nueva York: Harper and Row, 1957.
DAHL, R. Democracy and its critics. New Haven, CT: Yale University Press, 1989.

DAHL, R. La igualdad política. México, DF: FCE, 2008.

DOUBLEDAY, R.; WILSDON, J. (eds). Directions for scientific advice in Whitehall. Londres: University of Cambridge's Centre for Science and Policy; Science Policy Research Unit (SPRU) and ESRC STEPS Centre at the University of Sussex; Alliance for Useful Evidence; Institute for Government; and Sciencewise, 2013.

DOWNS, Anthony. An economic theory of democracy. Nueva York: Harper and Row, 1957.

ERICSSON, K. et. al. Expertise and expert performance. Cambridge: Cambridge University Press, 2006.

ESTLUND, D. Why not epistocracy. En: RESHOTKO, N. (ed.). Desire, identity and existence. Essays in the honor of T. M. Penner. Kelowna, BC:Academic Printing and Publishing, 2003.

ESTLUND, D. On Sunstein's infotopia. Theoria, v. 56, n. 119, p. 14-29, 2009.

FISCHER, F. Democracy and expertise: reorienting policy inquiry. Oxford: Oxford University Press, 2009.

FREY, B.; POMMEREHNE, W.; SCHNEIDER, F; GILBERT, G. Consensus and dissension among economist: an empirical inquiry. The American Economic Review, v. 74, n. 5, p. 986-994, 1984.

GALBRAITH, J. K. La economía del fraude inocente. La verdad de nuestro tiempo. Barcelona: Crítica, 2004. 
GARCÉ, A. Regímenes políticos de conocimiento: construyendo un nuevo concepto a partir de cambios seleccionados en políticas públicas del gobierno de Tabaré Vázquez (Uruguay, 2005-2009). Revista de Ciencia Política, v. 34, n. 2, p. 439-458, 2014.

GOLDMAN, A. Experts: which ones should you trust? En: GOLDMAN, A.;WHITCOMB, D. (eds). Social epistemology: essential readings. Oxford: Oxford University Press, 2011. GREPPI,Andrea. Concepciones epistémicas y concepciones doxásticas de la democracia. Eunomía. Revista en Cultura de la Legalidad, v. 4, p. 42-72, 2013.

HEAD, B. W. Reconsidering evidence-based policy: key issues and challenges. Policy and Society, v. 29, p. 77-94, 2010. HOLST, C. Why not epistocracy? Political legitimacy and 'fact of expertise'. En HOLST, C. (ed.). Expertise and democracy. Oslo: Centre for European Studies, University of Oslo, 2014a. HOLST, C. (ed.). Expertise and democracy. Oslo: Centre for European Studies, University of Oslo, 2014b.

HOLST, C. y MOLANDER, A. Epistemic democracy and the accountability of experts. En HOLST, C. (ed.). Expertise and democracy. Oslo: Centre for European Studies, University of Oslo, 2014.

INNES, J.; BOOHER, D. Collaborative polcymaking: governance through dialogue. En HAJER, M.; WAGENAAR, H. (eds). Policy analysis. Understanding governance in the network society. Cambridge: Cambridge University Press, 2003.

JASSANOF, S. Momentos constitucionales en el gobierno de la ciencia y la tecnología. En: AA.VV (T. Pérez Bustos \& M. Lozano Borda, Eds.). Ciencia, tecnología y democracia: reflexiones en torno a la apropiación social del conocimiento. Medellín: Colciencias y Universidad EAFIT, 2011.

KAPPEL, K. Factual disagreement and political legitimacy. En:HOLST, C. (ed). Expertise and democracy. Oslo: Centre for European Studies, University of Oslo, 2014.

KEARL, J. K.; POPE, C.; WHITING, G.; WIMMER, L. A confusion of economist? The American Economic Review, v. 69, n. 2, p. 28-37, 1979.

KING, W. F. H. Classical and foreign quotations, law terms and maxims, proverbs, mottoes, phrases, and expression in French, German, Greek, Italian, Latin, Spanish, and Portuguese. Londres: Whitaker and Sons, 1887.

LANDEMORE, H.; ELSTER, J. (eds). Collective wisdom: principles and mechanism. Cambridge: Cambridge University Press, 2014.

MOODIE, J.; HOLST, C. For the sake of democracy? The
European Commission's justifications for democratizing e xpertise. En HOLST, C. (ed.). Expertise and democracy. Oslo: Centre for European Studies, University of Oslo, 2014.

MORLINO, L. What is a 'good' democracy?. Democratization, v. 11, n. 5, p. 10-32, 2004.

MUNCK, G. Los estándares de la democracia: hacia una formulación de la cuestión democrática en América Latina. Journal of Democracy en Español, 22-41, 2010.

MUNCK, G. L.; VERKUILEN, J. Conceptualizing and measuring democracy: evaluating alternative indices. Comparative Political Studies, v. 35, n. 1, p. 5-34, 2002.

PEDERSEN, D. B. A dual justification for science-based policy-making. En HOLST, C. (ed.). Expertise and democracy. Oslo: Centre for European Studies, University of Oslo, 2014. PREWITT, K.; SCHWANDT,T.A.; STRAF, M. L. (eds). Using science as evidence in public policy. Washington, DC: National Academies Press, 2012.

ROSANVALLON, P. La legitimidad democrática: imparcialidad, reflexividad, proximidad. Buenos Aires: Manantial, 2009.

SABEL, C-F; ZEITLIN, J. Experimentalist governance. En: FAUR, L. (eds.). The Oxford Handbook of Governance. Oxford: Oxford University Press, 2012.

SAPIR, J. Economistas contra la democracia. Los intereses inconfesables de los fasos expertos de la economía. Barcelona: Ediciones B, 2004. SIBILIA, P. El hombre postorgánico. Buenos Aires: FCE, 2005. STEETS, J. Accountability in public policy partnerships. Nueva York: Palgrave Macmillan, 2010.

STEIN, E. et. al. (eds). La política de las políticas públicas. Washington, DC: BID, 2006.

STIGLITZ, J. Los felices noventa. La semilla de la destrucción. México, DF:Taurus, $2003 \mathrm{a}$.

Globalizations and its discontents. Nueva York: W. W.

Norton \& Company, 2003b.

The Stiglitz report: reforming the international monetary and financial systems in the wake of the global crisis. Nueva York: The New Press, 2010.

SUNSTEIN, C. Infotopia: how many minds produce knowledge. Oxford: Oxford University Press, 2006.

THOMPSON, D. Moral responsibility of public officials: the problem of many hands. American Political Science Review, v. 74, p. 905-916, 1980.

ZITO, A. Expertise and power. Environmental agencies operating in complex policy environments. En HOLST, C. (ed.). Expertise and democracy. Oslo: Centre for European Studies, University of Oslo, 2014.

\title{
Quality of democracy and factual disagreements. A partial exploration of the relation between citizens, politicians and experts
}

\author{
Abstract: \\ The crescent role of the knowledge and the act of experts in the contemporary democracies can be reached in three levels well defined: \\ 1) the distribution of the knowledge, the actors who know (experts and specialists) and their fit in the democratic legitimacy consist in \\ the epistemic level of democracy; 2) the role attributed to the knowledge, the expert and the rules of expertise in the democratic operation
}


characterizes the level of quality of democracy; and finally, 3) how States and governments use the expert knowledge to improve the democratic operation conform the level of agenda of the quality of democracy. This paper is located in the second level, between the philosophical normative and the empirical informative. This approach of the object is justified for two reasons: a) the philosophical discussion could go further in that the debates have access to systematic information and empirical validations, however, b) the empirical studies have, in my view, a limitation to its development, which is stanched by some imprecision in the conceptual definition of "expert" and his role in the democratic theory. The purpose of the paper is to analyze this topic.

Key words: quality of democracy, factual disagreement, experts, citizens.

\section{Qualidade da democracia e desacordos fáticos. Uma exploração parcial da relação entre cidadãos, políticos e expertos}

\section{Resumo:}

O crescente papel do conhecimento e o atuar dos expertos nas democracias contemporâneas podem ser abordados em três níveis bem delimitados: 1) a distribuição do conhecimento, os atores que conhecem (expertos e especialistas) e seu encaixe na legitimidade democrática constituem o nível epistêmico da democracia; 2) o papel que se atribui ao conhecimento, ao experto e às regras da expertise nos funcionamentos democráticos configura o nível da qualidade da democracia; y finalmente, 3) como usam os Estados e governos o conhecimento experto para melhorar os funcionamentos democráticos conforma o nível de agenda da qualidade da democracia. Este trabalho se situa no segundo nível, ou seja, entre o nível filosófico-normativo e o empírico-informativo. Este recorte do objeto de estudo está justificado por dois razões: a) a discussão filosófica poderia avançar mais na medida em que os debates tenham acesso a informação sistematizada e validações empíricas, no entanto, b) os estudos empíricos encontram, a meu modo de ver, um limitante a seu desenvolvimento, este está estancado por certa imprecisão na definição conceitual de "experto" e seu papel na teoria democrática. Este trabalho tem como propósito analisar este último assunto.

Palavras-chave: qualidade da democracia, desacordos fáticos, expertos, cidadãos. 
